\title{
Medicaid Policy on Sterilization - Anachronistic or Still Relevant?
}

Sonya Borrero, M.D., Nikki Zite, M.D., Joseph E. Potter, Ph.D., and James Trussell, Ph.D.

F emale sterilization, typically accomplished by means of tubal ligation, is a widely used method of contraception that is highly effective at preventing unintended pregnancy. Yet there appears to be unmet demand for the procedure in certain segments of the U.S. population. ${ }^{1}$ Specifically, low-income women and women from minority racial and ethnic groups may face substantial system-level barriers to obtaining a desired sterilization procedure. ${ }^{1}$ One such barrier is the federal policy regarding Medicaidfunded sterilizations. Although this policy was designed to protect vulnerable populations, we believe that it does not effectively fulfill that intention - in fact, it restricts the reproductive autonomy of the women it intends to serve. With the upcoming Medicaid expansions, the number of women affected by these barriers could increase substantially.

The use of tubal ligation as a contraceptive method increased dramatically during the 1960s and 1970s with the legalization of contraception, improved safety of laparoscopic techniques, and the creation of federally funded family-planning programs that subsidized the costs. During those years, numerous reports of coercive and nonconsensual sterilization of minority and poor women emerged, inciting a public outcry in which the government was accused of racist and classist applications of family-planning programs. In response, in 1976, the Department of Health, Education, and Welfare developed protective regulations and a standardized consent form for publicly funded sterilization procedures. These regulations prohibited sterilization of persons younger than 21 years of age or who were mentally incompetent or institutionalized. They also required a 72-hour waiting period before sterilization; in 1978, the waiting period was extended to 30 days from the time of written informed consent. Although these regulations apply to both women and men, female sterilization is significantly more common than male sterilization in low-income populations, and women often desire sterilization at a specific time - after childbirth - which makes the extended waiting period particularly problematic.

Because these policies have not changed since 1978, women requesting publicly funded sterilization must complete the "Consent to Sterilization" section of the Medicaid Title XIX form (Title XIX-SCF) at least 30 days and no more than 180 days before undergoing the procedure. In addition, a signed copy of the consent form must be available or verified at the time of the procedure. If the woman is undergoing emergency abdominal surgery or a premature delivery, the 30-day waiting period may be waived, but at least 72 hours must have elapsed between the consent and the procedure.

Although the policy was presumably well intentioned, there is evidence that the Medicaid consent process may not be capable of protecting vulnerable women by ensuring that truly informed consent is obtained. Although the consent form contains language designed to confirm the wom- an's understanding of the risks and benefits of the procedure, including the fact that the result is permanent, as well as information about the mandatory 30day waiting period, assessments of the form's readability indicate that it is overly complicated and its literacy level is too high for the average American adult. ${ }^{2}$ In one study assessing women's knowledge about sterilization after they had been given the Medicaid consent form for review, more than one third of respondents (34\%) answered incorrectly when asked about the permanence of sterilization. ${ }^{3}$ When a modified, low-literacy version of Title XIXSCF was compared with the current form in a randomized trial involving 200 women with Medicaid coverage, those who reviewed the modified form were more likely to know about the 30-day waiting period before the form is considered valid (a 24-percentagepoint difference between groups), that nonpermanent contraceptive options as effective as sterilization are available (an 8-percentage-point difference), and that the procedure is permanent (a 16-percentagepoint difference). ${ }^{3}$

Beyond concerns about the consent form, the waiting period and the need for the completed form to be transferred to the delivery unit pose logistic barriers for women who wish to undergo tubal ligation immediately after giving birth. Currently, among U.S. women sterilized within 2 years after delivery, more than $70 \%$ of procedures are done in the immediate postpartum period. Women report that requesting sterilization too late in pregnancy to fulfill 


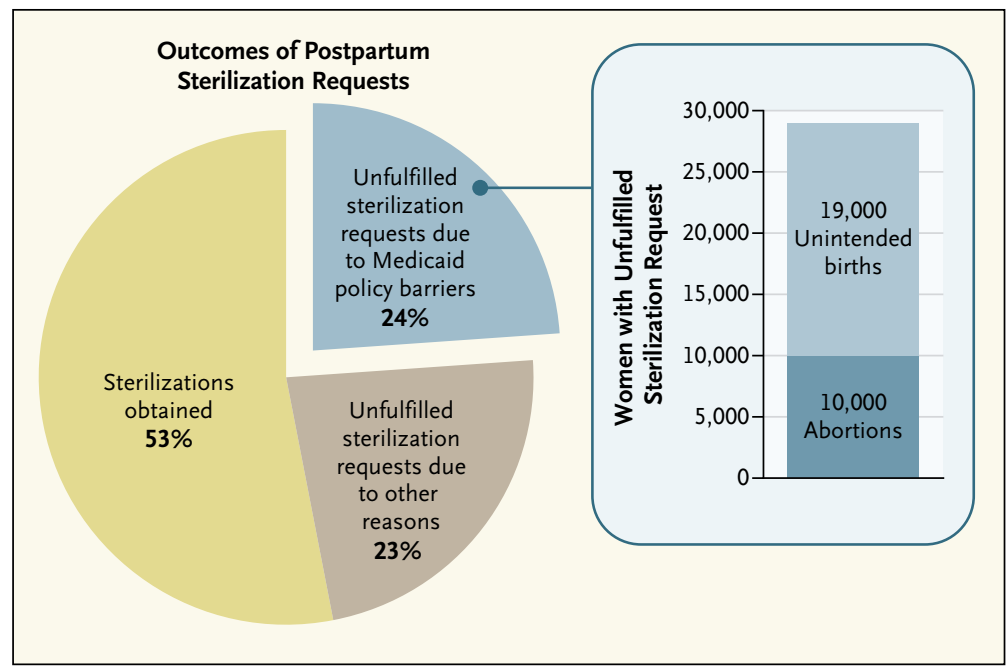

Estimated Annual Number of Requests for Postpartum Sterilization That Go Unfulfilled Because of Medicaid Policy Barriers, and Unintended Pregnancies in the Subsequent Year.

The pie chart shows the outcomes of the estimated 255,000 requests for federally funded, postpartum sterilizations made each year. In the subsequent year, an estimated 29,000 unintended pregnancies will occur among the 62,000 women with sterilization requests that are unfulfilled because of Medicaid policy barriers, costing taxpayers $\$ 215$ million annually. Estimates are based on a cost-analysis model described in Borrero et al. ${ }^{5}$

the 30-day waiting period, not having the form present at the time of delivery, or delivering before the mandatory waiting period had elapsed prevented them from having their request for sterilization fulfilled at the time of delivery. ${ }^{1}$ Moreover, because women with private insurance are not subject to the same regulations, the policy creates a two-tiered system of access, in which lowincome women may not be able to exercise the same degree of reproductive autonomy as their wealthier counterparts. Inequitable access is compounded by the fact that many Medicaid beneficiaries who do not receive a desired sterilization during the immediate postpartum period may miss their window of opportunity, since pregnancy-related Medicaid eligibility ends shortly after delivery. These issues recently prompted the American College of Obstetrics and Gynecology Committee on Health Care for Underserved
Women to recommend revising Medicaid policy to create fair and equitable access to sterilization procedures. ${ }^{1}$

Preventing women from obtaining a desired sterilization puts them at high risk for unintended pregnancy. In one study, $47 \%$ of women who requested but did not receive a postpartum sterilization became pregnant during the first year after delivery over twice the rate of pregnancies in women who did not request sterilization. ${ }^{4}$ Unfortunately, unintended pregnancy remains a prevalent and substantial issue pregnancies are associated with numerous adverse health and social consequences for women and their families. In addition, unintended pregnancy is costly for society, with direct annual public costs of billions of dollars. Reducing barriers associated with Medicaid policy regarding sterilization may be one in the United States, since such approach to making a dent in the stubbornly high rate of unintended pregnancy and the high costs associated with it. In a recent cost analysis, we found that Medicaid-policy-related barriers lead to approximately 62,000 unfulfilled requests for postpartum sterilization annually, resulting in an estimated 10,000 abortions and 19,000 unintended births in the subsequent year, at a public cost of $\$ 215$ million (see chart). ${ }^{5}$

Discussions about modifying federal policy on sterilization need to be thoughtful and sensitive to the fact that there is a larger social and historical context in which the reproductive behavior of low-income and minority women has commonly been negatively stereotyped and their fertility less valued than that of other women. Unfortunately, as recent allegations that nearly 150 incarcerated women in California underwent coercive sterilizations between 2006 and 2010 attest, protection for vulnerable women is still needed. However, given the high rate of unintended pregnancy, enhancing access to each woman's contraceptive method of choice is also critical. An ideal Medicaid policy or process would strive to meet these two goals by ensuring patient comprehension and facilitating access to sterilization for women who have made an informed decision to undergo the procedure.

To this end, we believe that the current Medicaid Title XIX sterilization consent form needs to be either redesigned, in order to present the pertinent information in an easier-to-read, userfriendly format, or ideally replaced altogether by a validated decision-support tool that can more effectively ensure an informed decision-making process. 
In addition, we believe that the 30-day mandatory waiting period is excessive and should be shortened considerably or eliminated - a change that could also address the problem of failed transfer of the completed federal document to the delivery unit.

Although the principles behind the Medicaid policy remain relevant, it is in dire need of modification. Measures to promote informed decision making regarding sterilization, rather than stringent and restrictive regulations, can simultaneously protect vulnerable populations and allow women to reduce their risk of unintended pregnancy. Thus, revisiting and amending sterilization policy represents an opportunity to honor women's reproductive autonomy, create more equitable access to sterilization, save a substantial amount of health care dollars, and prevent unintended pregnancy.

Disclosure forms provided by the authors are available with the full text of this article at NEJM.org.

From the Division of General Internal Medicine, University of Pittsburgh School of Medicine, and the Center for Health Equity, Research, and Promotion, Veterans Affairs Pittsburgh Healthcare System - both in Pittsburgh (S.B.); the Department of Obstetrics and Gynecology, University of Tennessee Graduate School of Medicine, Knoxville (N.Z.); the Population Research Center, University of Texas at Austin, Austin (J.E.P.); the Office of Population Research, Princeton University, Princeton, NJ (J.T.); and the Hull York Medical School, Hull, United Kingdom (J.T.).

1. Committee on Health Care for Underserved Women. Access to postpartum sterilization.
Committee opinion no. 530. Washington, DC: American College of Obstetricians and Gynecologists, July 2012 (http://www.acog.org/ Resources_And_Publications/Committee_ Opinions/Committee_on_Health_Care_ for_Underserved_Women/Access_to_ Postpartum_Sterilization).

2. Zite NB, Philipson SJ, Wallace LS. Consent to Sterilization section of the MedicaidTitle XIX form: is it understandable? Contraception 2007;75:256-60.

3. Zite NB, Wallace LS. Use of a low-literacy informed consent form to improve women's understanding of tubal sterilization: a randomized controlled trial. Obstet Gynecol 2011;117:1160-6.

4. Thurman AR, Janecek T. One-year followup of women with unfulfilled postpartum sterilization requests. Obstet Gynecol 2010; 116:1071-7.

5. Borrero S, Zite N, Potter JE, Trussell J, Smith K. Potential unintended pregnancies averted and cost savings associated with a revised Medicaid sterilization policy. Contraception 2013;88:691-6.

DOI: 10.1056/NEJMp1313325

Copyright @ 2014 Massachusetts Medical Society.

\title{
Mom at Bedside, Appears Calm
}

\author{
Suzanne Koven, M.D.
}

W e carry a nylon lunch bag everywhere we go, royal blue with purple trim, containing two plastic syringes, each preloaded with $5 \mathrm{mg}$ of liquid Valium, plus packets of surgical lubricant and plastic gloves. At the first sign of blinking or twitching, we lay him on his left side, tug down the elastic waist of his pants, part his small buttocks, and insert the gooped-up tip. Within moments, the motion stops, as if an engine has been switched off. Then he falls into a deep sleep. When he relaxes, so do we.

He's 5 years old, the first time. Our babysitter takes him to a pizza place for lunch. He laughs mid-slice, blinks his eyes several times, slumps to the floor, and climbs back onto his chair. She hesitates - what was that? and then calls 911 . She pages me.
I keep the message stored in my beeper, periodically daring myself to relive my first reading of it.

I meet them in the ER at the community hospital near our home, showily flashing my downtown hospital ID tag. Soon my husband rushes in, wearing the ID from his downtown hospital. All the tests are negative, they say. Bring him back if something else happens.

Something else happens. The next day, I skip work and keep him home from school. He sits happily in front of cartoons while I pace and polish, pace and fold. Maybe the babysitter overreacted, I reason. Maybe he's just a goofy kid. The moment I stop watching him, he cries, "Look, Mommy! Look what my hand can do!"

Downtown. No mistaking it this time. Grand mal, big and bad, right on the gurney. Lumbar puncture. MRI. All negative. Before we go home, the neurologist asks if we have further questions. "Just one," I say. "What do we do if he does it again?" The neurologist seems surprised. His raised eyebrows silently ask, "Aren't you both doctors?" He hands us a pamphlet.

Dilantin. Chewable yellow triangles three times a day. Triangles to first grade and the beach and day camp and a sleepover. The other kid has cochlear implants. "Don't worry," his mother says, accepting my baggie of pills. "My kid comes with instructions, too." We become members of an exclusive club no one wants to join.

One day, almost exactly a year later, the school nurse calls. "It's been 10 minutes and it's not 